\title{
Two-thumb-encircling advantageous for lay responder infant CPR: a randomised manikin study
}

\author{
Jeffrey L Pellegrino, ${ }^{\oplus}$ David Bogumil, ${ }^{2}$ Jonathan L Epstein, ${ }^{3}$ Rita V Burke ${ }^{2}$
}

${ }^{1}$ Health Sciences, Aultman College of Nursing and Health Sciences, Canton, Ohio, USA ${ }^{2}$ Keck School of Medicine, University of Southern California, Los Angeles, California, USA

${ }^{3}$ American Red Cross, Washington, District of Columbia, USA

\section{Correspondence to}

Dr Jeffrey L Pellegrino, Health Sciences, Aultman College of Nursing and Health Sciences, Canton, OH 44710, USA; jeffrey.pellegrino@ aultmancollege.edu

Received 30 January 2018 Revised 4 June 2018 Accepted 22 June 2018 Published Online First 14 July 2018

\section{Linked}

- http://dx.doi.org/10.1136/ archdischild-2018-315756

Check for updates

(c) Author(s) (or their employer(s)) 2019. No commercial re-use. See rights and permissions. Published by BMJ.

To cite: Pellegrino JL,

Bogumil D, Epstein JL, et al. Arch Dis Child

2019:104:530-534.
ABSTRACT
Objective Paediatric health providers and educators influence infant mortality through advocacy and training within families and communities. This research sought to establish the efficacy and training of two-finger versus two-thumb-encircling techniques for lone responder infant chest compressions with ventilations in initially trained infant caregivers.

Design This is a randomised, cross-over educational intervention assessed on instrumented manikins using the 2015 guideline measures of quality infant cardiopulmonary resuscitation (CPR). Additional subjective data on the experience were collected through self-reporting.

Setting Non-healthcare community organisations and secondary school classrooms.

Participants Fourteen years or older, fluent in English and had not taken infant CPR in the last 5 years.

Interventions Groups of eight participants were randomised to learn one technique, practised and then tested for $8 \mathrm{~min}$. After a 30 min rest, the group repeated the process using the other technique.

Main outcome measures Mean chest compression depth and rate, compression fraction, and correct hand position; tiredness and pain as reported by the caregiver. Results The two-thumb-encircling technique achieved a deeper mean compression depth over the $8 \mathrm{~min}$ period $(2.0 \mathrm{~mm}, \mathrm{p}<0.01)$, closer to the minimum recommendation of $40 \mathrm{~mm}$; the two-finger technique achieved higher percentages of compression fraction and complete recoil. Caregivers preferred the two-thumb technique (64\%), and of these $70 \%$ had long fingernails. Conclusions The two-thumb-encircling technique improved compression depth, over an 8 min scenario, and was preferred by caregivers. This adds to the existing literature on the advantages of two-thumb-encircling as a technique for lone and team infant CPR, which counters current guidelines.

\section{INTRODUCTION}

Healthcare professionals can improve infant mortality rates ${ }^{12}$ by providing awareness/training on quality cardiopulmonary resuscitation (CPR) among caregivers for out-of-hospital cardiac arrest (OHCA), starting with paediatricians and resuscitation organisations. ${ }^{3-5}$ Implementation of a successful evidence-based practice (EBP) requires balance of outcomes evidence, wisdom of experts and acceptance of those who are asked to use it. Recognising the diversity of infant caregivers as end users of CPR education contributes to the recommendation of implementing resuscitation guidelines in OHCA. ${ }^{6}$

\section{What is already known on this topic?}

Previous studies on healthcare providers demonstrate that the two-thumb chest compression technique is preferred by healthcare responders and best meets the current resuscitation guidelines.

- Lone and/or lay responder cardiopulmonary resuscitation efficacy is important to reduce infant mortality, and training those in closest proximity to infants increases the odds of response.

- Reducing complexity and barriers to care (ie, simple and easy compressions) increases willingness to help in resuscitation.

\section{What this study adds?}

- This was an observation of initially trained lay responders', specifically infant caregivers', ability to perform and preference for two-thumb chest compression with ventilations over a clinically relevant $8 \mathrm{~min}$ period.

- This provides community education evidence to add to previous research on paediatric professionals' advocacy of training target populations with the most efficient techniques.

Mechanically, CPR techniques strive to meet five quality components: chest compression rate (CR) (100-120 min), chest compression depth (CD) (at least one-third of the anterior-posterior thoracic diameter, minimum of $40 \mathrm{~mm}$ ), full chest recoil, minimum interruptions in chest compressions and avoiding excessive ventilation. ${ }^{7}$ The 2015 European Resuscitation Guidelines describe two different compression techniques: two-thumb-encircling technique (TT) for two responders and the two-finger technique (TF) for a lone responder. ${ }^{8}$ No evidence, however, exists for either technique on the critical outcomes of time to return of spontaneous circulation, survival rates or neurological injury; and only low-quality evidence on the critical outcome of improved perfusion and gas exchange during CPR, which identified higher blood pressure generation with the TT versus TF technique. On the question of how either technique affects the responders, low-quality evidence suggested TT is less tiring. ${ }^{9}$ All of the studies that contributed to the Consensus on Science and Treatment Recommendations used healthcare providers as compressors. ${ }^{10}$ 
No studies exist that describe the initial training of infant caregivers using TF compared with TT, or TT for the lone responder. Existing studies use periods of 1-5 min, which does not represent the 8-10 min of actual CPR needed prior to emergency medical services (EMS) taking over. ${ }^{11}$ We hypothesise that TT performs better than TF in quantitative and qualitative measures. The following are the aims of this study on initially trained infant caregiver:

1. Describe the performance of TT and TF techniques over $8 \mathrm{~min}$.

2. Identify the factors or characteristics that lead to efficacy.

3. Identify which technique(s) might be advantageous for teaching and effectiveness.

The primary outcomes included CD ( $\mathrm{mm}), \mathrm{CR}$ (per minute), percentage of compressions with adequate rate (100-120 per minute), compression fraction (CF, percentage of time with compressions), percentage with correct hand position, correctly released compression and total hands-off time (in seconds) as measured by the PC SkillReporting System (Laerdal, Stavanger, Norway). The inclusion criteria included using the taught TT or TF techniques, as observed by the researchers. The secondary outcomes included self-rating of pain, fatigue and preference.

\section{METHODS}

We conducted a prospective, randomised, cross-over experimental study of TT and TF compressions on initially trained infant caregivers who were recruited from schools and community health services in a mid-western city (USA). Eligibility criteria included no infant CPR training in the previous 5 years, non-healthcare provider, 14 years of age or older, with two hands, and with no acute pain or injury in the upper body. Per the sample size calculation methodology and the estimate of sigma from Boldingh et al, ${ }^{12}$ an a priori power analysis resulted in a minimum sample size of $n=24$ required to have an $80 \%$ chance of detecting a meaningful difference (d) of 10 compressions per minute differential between the two compression methods using a pooled SD of 16.6 .
Per protocol, the participants learnt about infant mortality and the value of CPR prior to agreeing to participate and completing the presurvey. The researchers oriented the participants to a 3-month-old infant-sized manikin (Laerdal Resusci Baby QCPR, Laerdal) resting on standard-height tables $(71 \mathrm{~cm})$. For randomisation, a participant drew a chip labled with either TT or TF technique from a bag for the group to start with; followed by an instruction that lasted approximately $4 \mathrm{~min}$, consisting of hand(s) or finger placements and ventilation skills. Using visual feedback and coaching to demonstrate guideline compliances, participants then practised approximately 6 more minutes and before a $10 \mathrm{~min}$ break.

After removing the monitor, the first four participants began the $8 \mathrm{~min}$ CPR scenario. The researchers read a statement at 2 min intervals to provide a sense of time in context (see figure 1). The second set of four participants then practised and completed the $8 \mathrm{~min}$ scenario. After completion of the initial technique, the group took $30 \mathrm{~min}$ to learn about the factors that affect infant mortality, providing a washout period, then repeated the learning sequence for the second technique. A postsurvey and discussion of personal satisfaction with each technique and the likelihood of performing either during an emergency were conducted.

Nine 90 min group sessions were conducted, with group size of two to eight individuals (mean $=5$ ). We calculated the mean, median and SD of the demographic variables age, height, weight, finger span and grip strength. Age was also displayed as a category due to its non-normal distribution. Categorical age and gender were described in per cent and frequencies. Immediately after each technique, the participants self-identified where any pain existed and rated their overall pain level on a scale of $0-10$, with 10 representing the worst pain ever experienced and 1 a noticeable feeling of discomfort.

We used linear regression to estimate the differences in the effects of continuous outcomes between the techniques, and logistic regression to measure the ratio of the effects of dichotomous variables. We checked the linear regression models for linearity, normality and homoscedasticity (the error being

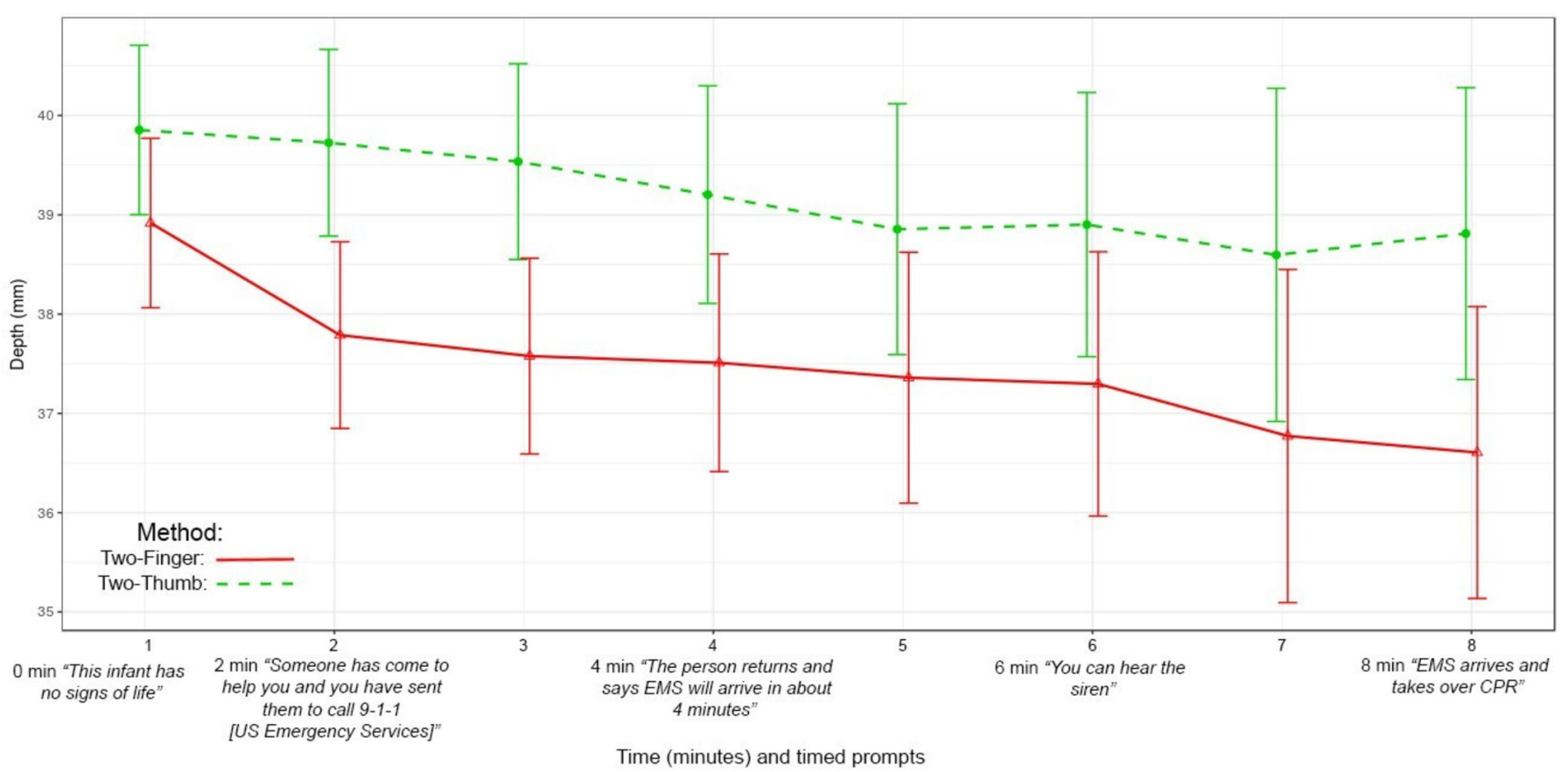

Figure 1 Fatigue according to methods with timed prompts. CPR, cardiopulmonary resuscitation. 


\begin{tabular}{|c|c|c|c|}
\hline Continuous variables & Mean (SD) & Median & $\mathrm{IQR}^{*}$ \\
\hline Age (years) & $23.1(11.4)$ & 17.0 & 11.0 \\
\hline Height $(\mathrm{cm})$ & $160.4(9.6)$ & 160.0 & 12.7 \\
\hline Weight (kg) & $71.9(19.2)$ & 68.1 & 26.3 \\
\hline Finger span $(\mathrm{cm})$ & $19.4(1.6)$ & 19.7 & 2.5 \\
\hline Grip (kg) & $28.1(7.1)$ & 27.6 & 6.8 \\
\hline Categorical variables & $\%(n)$ & & \\
\hline \multicolumn{4}{|l|}{ Age distribution (years) } \\
\hline $16-18$ & $69.0(29)$ & - & - \\
\hline $19-25$ & $4.8(2)$ & - & - \\
\hline $26-45$ & $21.4(9)$ & - & - \\
\hline $46-60$ & $4.8(2)$ & - & - \\
\hline Gender: female & $95.2(40)$ & - & - \\
\hline
\end{tabular}

${ }^{*}$ IQR (25th-75th percentile).

equal across the values of the independent variable) of residuals using scatter and locally weighted scatterplot smoothing plots of predicted values against the residuals and the histograms of the residuals. If these assumptions were violated, two-sided $\mathrm{p}$ values were calculated using non-parametric rank-based tests to compare the medians. Two-sided Wald $\mathrm{p}$ values were generated for all effect estimates and a $p$ value $<0.05$ was used to determine statistical significance. The models used a random intercept term to account for individual-level pairing due to the cross-over design. Additionally, given the self-reported preferred technique, differences in responder attributes based on preference were estimated. All analyses were conducted on R (V.3.1.1).

\section{RESULTS}

Sample characteristics

Fifty-four individuals participated in January 2017; however, we excluded 12 individuals, 4 because of data recording issues and 8 because they did not encircle the chest during TT. We used data from the remaining 42 participants in the analysis (table 1). Examination of the demographic data from both samples showed no significant differences between the groups. Most of the sample being between 16 and 18 years (table 1 ) leaves it right-skewed, with a minimum age of 16 and a maximum of 60 , and a median of 17 . Women comprised majority of the sample (95.2\%). Participants' self-reported height averaged $160.4 \mathrm{~cm}$ and their weight averaged $71.9 \mathrm{~kg}$. The mean finger span, from extended pinky fingertip to the tip of the thumb, was $19.3 \mathrm{~cm}$, and the average grip strength was $28.1 \mathrm{~kg}$.

\section{Measures of effect by compression technique}

On average, participants using the TT technique demonstrated a $2.0 \mathrm{~mm}(\mathrm{p}<0.01)$ deeper $\mathrm{CD}$ over the $8 \mathrm{~min}$ period (table 2 , figure 1$)$. The TF data showed improved CF ( $2 \%$ greater among TF, $p=0.03)$ and correctly released compression $(21.6 \%$ greater among TF, $\mathrm{p}<0.01$ ). For self-reported measures, the techniques did not show statistically significant differences in reported tiredness but almost reached significance in self-reported pain $(\mathrm{p}=0.07)$.

\section{Learner attributes by technique preference}

Participants reported preferring to use TT (64\%). Based on preferred compression technique, only non-significant differences existed by participant physical characteristics (table 3). Those who preferred the TT technique averaged a finger span of $1.1 \mathrm{~cm}$ larger than those who preferred the TF technique; however, this was not a statistically significant difference $(\mathrm{p}=0.33)$. The participants who preferred the TF technique were $0.5(p=0.06)$ times as likely to have long fingernails (extending beyond the fingertip).

\section{Self-reported discomfort/pain}

All participants reported no current/existing pain in the upper extremities at the start of the study. The location of pain differed between the techniques. With the TF technique, $85 \%$ of pain was in the hands (fingers, knuckles), with an additional $12 \%$ in the arms (wrists, upper extremity). Pain associated with the TT technique occurred $62 \%$ of the time in the hands, $26 \%$ in the back and $10 \%$ in the arms.

\section{DISCUSSION}

Limited evidence currently exists as to the optimal infant compression technique; this study reports on the objective

Table 2 Measures of effects by compression technique $(n=42)$

\begin{tabular}{|c|c|c|c|c|c|c|c|}
\hline & \multicolumn{2}{|c|}{ CPR technique mean value } & \multicolumn{2}{|c|}{ CPR technique median value } & \multicolumn{2}{|c|}{ Difference* $^{*}$} & \multirow[b]{2}{*}{$P$ valuest } \\
\hline & TT & TF & TT & TF & Mean & Median & \\
\hline \multicolumn{8}{|l|}{ Technique measures } \\
\hline Compression depth (mm) & 39.8 & 37.8 & 40.0 & 38.0 & 2.0 & 2.0 & $<0.01$ \\
\hline Mean rate (compressions per minute) & 114.1 & 116.1 & 114.0 & 117.5 & -2.0 & -3.5 & 0.31 \\
\hline Deep enough compressions (\%) & 52.2 & 51.2 & 55.5 & 46.5 & $1.4 \ddagger$ & 9.0 & $0.78 \ddagger$ \\
\hline Compressions with adequate rate (\%) & 59.6 & 57.5 & 68.0 & 57.0 & $3.7 \ddagger$ & 11.0 & $0.45 \ddagger$ \\
\hline Compression fraction (\%) & 69.6 & 73.3 & 72.0 & 74.0 & -3.7 & -2.0 & $0.03 \S$ \\
\hline Correct hand position (\%) & 82.8 & 85.1 & 96.0 & 93.5 & -2.3 & 2.5 & $0.39 \S$ \\
\hline Correctly released compression (\%) & 53.2 & 74.8 & 47.5 & 88.0 & -21.6 & -40.5 & $<0.01$ \\
\hline Average hands-off time (s) & 30.2 & 27.8 & 28.8 & 28.8 & 2.4 & 0.0 & $0.30 \S$ \\
\hline No flow time (s) & 130.7 & 128.1 & 129.6 & 124.8 & 2.6 & 4.8 & $0.70 \S$ \\
\hline \multicolumn{8}{|l|}{ Self-reported measures } \\
\hline Tiredness score & 3.7 & 3.9 & 3.0 & 3.5 & -0.2 & -0.5 & 0.98 \\
\hline Pain score & 4.9 & 5.8 & 4.0 & 6.0 & -0.9 & -2.0 & 0.07 \\
\hline
\end{tabular}

* Matched measure of effect.

†Two-sided Wald test $p$ value, unless specified.

$¥$ Adjusting for ordering of training, due to change in estimates greater than $20 \%$.

$\S$ Rank-based $p$ value, due to violation of regression assumptions.

CPR, cardiopulmonary resuscitation; $\mathrm{TF}$, two-finger; $\mathrm{TT}$, two-thumb. 
Table 3 Attributes by technique preference $(n=42)$

\begin{tabular}{lcclll}
\hline & \multicolumn{3}{l}{ Preferred technique } & & \\
\cline { 2 - 3 } & TT & TF & Difference (SE) & P values* \\
\hline Continuous & & & & \\
Age (years) & 22.3 & 24.6 & $-2.3(-)$ & $0.66 \dagger$ \\
Height $(\mathrm{cm})$ & 160.4 & 160.4 & $-0.1(3.1)$ & 0.98 \\
Weight $(\mathrm{kg})$ & 69.0 & 77.0 & $-8.0(-)$ & $0.09 \dagger$ \\
Finger span $(\mathrm{cm})$ & 19.8 & 18.8 & $1.1(-)$ & $0.33 \dagger$ \\
Average grip $(\mathrm{kg})$ & 27.6 & 28.9 & $-1.3(2.3)$ & 0.58 \\
\hline Categorical & & & Prevalence ratio & P values \\
\hline Long fingernails & $70.0 \%$ & $35.0 \%$ & $0.5(0.4)$ & 0.06 \\
\hline
\end{tabular}

*Two-sided Wald test $p$ value, unless specified.

tRank-based $p$ value.

¥Restricted to 30 individuals with non-missing nail data.

$\mathrm{TF}$, two-finger; $\mathrm{TT}$; two-thumb.

differences and preferences of infant caregivers. CD showed the largest and perhaps most clinically relevant difference, with TT being $2 \mathrm{~mm}$ closer to the $40 \mathrm{~mm}$ minimum than TF, according to Sutton et al..$^{13}$ Correctly released compressions favoured TF (21.6\% difference) and might be attributed to the increased pain of TF and relief from the pressure. As a binary, correctly released or not, we cannot assign benefit because release did occur in TT, but it is unknown to what extent.

Although ventilation volume could not be measured, we observed participants adjusting their position relative to the infant (ie, moving around the corner of the table) during TT, which may contribute to lower CF. Insignificant differences existed in the mean CR, percentage of deep enough compressions, adequate rate percentage and correct hand position percentage between the two techniques.

Efficiency or effectiveness of the techniques could not be attributed to age, height, weight, finger span or grip strength. Participants reported preferring to use TT (64\%), 70\% of whom had fingernails extending beyond the fingertips. This characteristic has not been previously reported in the literature, most likely because healthcare professionals would be expected to have shorter fingernails for infection control. The researchers observed long fingernails to negatively affect the ability/ effectiveness of TF and the willingness to learn. Individuals attempting to follow the TF training or just-in-time directions from a dispatcher may be less effective with CPR, unwilling/ unable due to long fingernails.

The randomisation of the initial technique and the consistency of the learning protocol in time reduced bias towards one technique over the other. In asking the participants to rate the experience of each technique, tiredness did not appear significantly different, but experience of pain during or immediately afterwards was rated higher in TF. In observing the participants, the table height may contribute to discomfort as people tended to be hunched over more with the TT technique.

Overall, individuals performed closer to the current guidelines than the infant CPR instructors in the Martin et al study. ${ }^{14}$ This observation may be attributed to the use of a visual feedback during practice. ${ }^{15}{ }^{16}$ We observed that the TT technique produced better CDs relative to the TF technique, regardless of fatigue, confirming Udassi et al's assertion of TT being superior over time. $^{17}$

Educators need to address all quality CPR components to assure adequate perfusion in future curriculum. Both techniques failed to achieve the $40 \mathrm{~mm}$ CD over an $8 \mathrm{~min}$ resuscitation period. The decline of CD over time suggests that compressors change when available at least every $2 \mathrm{~min}$ or when fatigued. Neither technique in the single responder scenario worked ideally; health educators need to address educational effectiveness to increase CF and full release in the TT.

\section{Limitations}

Recruitment of participants focused on potential infant caregivers from secondary schools and community participants, yielding a high percentage of women, which does not generalise to the US population. However $<20 \%$ of families have fathers as primary caregivers with working mothers, ${ }^{18}$ and $>94 \%$ of childcare providers are female. ${ }^{19}$ The sample also represents those choosing to be trained from the community.

Martin et $a l^{20}$ identified that manikins that allowed for $>40 \mathrm{~mm} \mathrm{CD}$, like the ones used with a maximum of $43 \mathrm{~mm}$, promoted deeper compressions closer to guidelines. This may partially explain why these participants were closer to guidelines than professionals in previous studies; those studies did not report on manikin design. Ventilations were not reported because data could not be validated, leaving CD and rate as primary reportable data, which leaves additional research to be completed.

We chose to use a group format while recording data to simulate face-to-face classroom experience, and this limited the ability to measure learners' readiness to apply the skills in a stressed situation. Future studies on retention or stress on a single responder compression technique would add valuable information for educational effectiveness.

The height of the infant from the floor may affect subjective or objective data based on the height of the responder and the need to lean over to make the technique effective. Responders may have to perform CPR under different conditions, such as on the floor, changing tables or other heights. Self-modification of TT without encircling ${ }^{21} 22$ should be examined for future lay responder training as it may represent a compromise between these two techniques.

To implement EBP, health educators must balance outcomes evidence, expert opinion and stakeholder willingness. TT showed significantly deeper compressions over the duration of $8 \mathrm{~min}$ and is reasonable to be learnt in a community, lone responder, level course. Based on the observed results, lay responders learnt a compression technique through practice on a feedback manikin and an instructor coaching in $10 \mathrm{~min}$ to demonstrate guideline-level skills, but failed to demonstrate CD, CF and recoil over an $8 \mathrm{~min}$ period in either TF or TT. Participants learnt the second technique in approximately $4 \mathrm{~min}$ because of prior practice with ventilations. As most infant cardiovascular collapse stems from asphyxia, additional studies that include ventilation measurements would add to the evidence of the TT efficacy in the infant caregiver population. Teaching TT in a single lay responder programme deviates from the current basic life support curriculum, and necessitates a reorientation of instructors and curriculum designers to a growing body of evidence and updating of educational materials. This study also adds knowledge on participants' experience of increased pain with TF and their preference for TT, while considering the effects of other physical characteristics. Ultimately, the Chain of Survival Behaviours ${ }^{6}$ relies on individual decisions to act to save a life by effective CPR. Effectiveness with regard to CD, CF and release is tied to both the clinical technique and lay responders' individual characteristics or situation with other responders. Preference, which may be related to comfort or willingness to use 
a technique, is at the individual's discretion and needs to be accounted for by advocates.

\section{CONCLUSION}

Potential lay responders learning infant CPR preferred TT over a realistic $8 \mathrm{~min}$ demonstration period. The TT technique was significantly associated with improved $\mathrm{CD}$, closer to the $40 \mathrm{~mm}$ minimum over the whole duration. This research also identified long fingernails factoring into the effectiveness and preference of responders. Curriculum designers and training organisations must consider the value and additional educational needs to effectively use TT in a lone responder situation.

Acknowledgements The authors would like to acknowledge Access Health Stark County and Canton City Schools for recruiting the participants, Aultman Hospital Birth Center Educator Sue Augustine for providing healthy infant information, future doctor Brian Miller for counselling on the methodology and statistical approach, Anna Knapski, Macy Hawk and Allison Barnes for assistance on data collection, and the American Red Cross Scientific Advisory Council for peer review.

Contributors JLP takes responsibility for the integrity of the work as a whole, including data collection, initial draft and revisions of the manuscript. DB provided substantial contributions to the analysis and interpretation of data. JLE, JLP and RVB provided substantial contributions to the conception and design of the work, as well as interpretation and reporting. All authors had access to all original data, participated in the drafting, approved the current version and agreed to be accountable for all aspects of the work.

Funding The authors have not declared a specific grant for this research from any funding agency in the public, commercial or not-for-profit sectors.

Competing interests None declared.

Ethics approval The Aultman Hospital Institutional Review Board (Canton, Ohio, USA) approved the protocols in January 2017.

Provenance and peer review Not commissioned; externally peer reviewed.

\section{REFERENCES}

1 U.S. Department of Health and Human Services. Midcourse Review: LHIs | Healthy People 2020. 2017 https://www.healthypeople.gov/2020/data-search/midcoursereview/hi (accessed 17 Oct 2017).

2 de Lucas N, Rodríguez-Núñez A, Van de Voorde P, et al. Witnesses, bystanders and outcome in paediatric out-of-hospital cardiac arrest. Resuscitation 2016;106:e21.

3 Citizen CPR Foundation. Citizen CPR Foundation- About CCPRF. 2016 https://www. citizencpr.com/ehome/200262/about/ (accessed 23 Apr 2017).

4 Frush K. Preparation for emergencies in the offices of pediatricians and pediatric primary care providers. Pediatrics 2007;120:200-12.

5 COMMITTEE ON HOSPITAL CARE and INSTITUTE FOR PATIENT- AND FAMILYCENTERED CARE. Patient- and family-centered care and the pediatrician's role. Pediatrics 2012;129:394-404.
6 International Federation of Red Cross Red Crescent Societies. International first aid and resuscitation quidelines 2016. Geneva, Switzerland: International Federation of Red Cross Red Crescent Socieites, 2016. http://www.ifrc.org/Global/Publications/ Health/First-Aid-2016-Guidelines EN.pdf.

7 Atkins DL, Berger S, Duff JP, et al. Part 11: Pediatric Basic Life Support and Cardiopulmonary Resuscitation Quality: 2015 American Heart Association Guidelines Update for Cardiopulmonary Resuscitation and Emergency Cardiovascular Care. Circulation 2015;132:S167-75.

8 Maconochie IK, Bingham R, Eich C, et al. European Resuscitation Council Guidelines for Resuscitation 2015: Section 6. Paediatric life support. Resuscitation 2015:95:223-48.

9 International Liaison Committee on Resuscitation. NRP 605. volunteer.heart.org. 2015 https://volunteer.heart.org/apps/pico/Pages/PublicComment.aspx?q=605 (accessed 14 May 2018)

10 Wyllie J, Bruinenberg J, Roehr CC, et al. European Resuscitation Council Guidelines for Resuscitation 2015: Section 7. Resuscitation and support of transition of babies at birth. Resuscitation 2015:95:249-63.

11 National Fire Protection Agency (NFPA). NFPA 1720: Standard for the Organization and Deployment of Fire Suppression Operations, Emergency Medical Operations and Special Operations to the Public by Volunteer Fire Departments. National Fire Protection Agency 2014 http://www.nfpa.org/codes-and-standards/all-codes-andstandards/list-of-codes-and-standards? mode $=$ code $\&$ code $=1720 \&$ tab $=$ editions

12 Boldingh AM, Jensen TH, Bjørbekk AT, et al. Rescuers' physical fatigue with different chest compression to ventilation methods during simulated infant cardiopulmonary resuscitation. J Matern Fetal Neonatal Med 2016;29:3202-7.

13 Sutton RM, French B, Niles DE, et al. 2010 American Heart Association recommended compression depths during pediatric in-hospital resuscitations are associated with survival. Resuscitation 2014;85:1179.

14 Martin PS, Kemp AM, Theobald PS, et al. Do chest compressions during simulated infant CPR comply with international recommendations? Arch Dis Child 2013;98:576-81.

15 Cheng A, Brown LL, Duff JP, et al. Improving cardiopulmonary resuscitation with a CPR feedback device and refresher simulations (CPR CARES Study): a randomized clinical trial. JAMA Pediatr 2015;169:137.

16 González-Salvado V, Fernández-Méndez F, Barcala-Furelos R, et al. Very brief training for laypeople in hands-only cardiopulmonary resuscitation. Effect of real-time feedback. Am J Emerg Med 2016;34:993-8.

17 Udassi S, Udassi JP, Zarusky AL, et al. Decay in quality of chest compressions with two fingers technique during lone rescuer infant manikin CPR. Crit. Care Med 2007;35:A6.

18 Laughlin L. Who's Minding the Kids? Child Care Arrangements. 2013. Washington, D C, 2011. https://www.census.gov/prod/2013pubs/p70-135.pdf.

19 Bureau of Labor Statistics. Household Data Annual Averages 11 Employed persons by detailed occupation, sex, race, and Hispanic or Latino ethnicity. Washington, D.C 2012. https://www.bls.gov/cps/aa2012/cpsaat11.pdf. (accessed 17 Oct 2017).

20 Martin PS, Kemp AM, Theobald PS, et al. Does a more "physiological" infant manikin design effect chest compression quality and create a potential for thoracic overcompression during simulated infant CPR? Resuscitation 2013;84:666-71.

21 Smereka J, Kasiński M, Smereka A, et al. The quality of a newly developed infant chest compression method applied by paramedics: a randomised crossover manikin trial. Kardiol Pol 2017;75:589-95.

$22 \mathrm{Na}$ JU, Choi PC, Lee HJ, et al. A vertical two-thumb technique is superior to the twothumb encircling technique for infant cardiopulmonary resuscitation. Acta Paediatr 2015;104:e70-5. 